\title{
DOA and Range Estimation using a Uniform Linear Antenna Array without a Priori Knowledge of the Source Number
}

\author{
Jianzhong Li, Yide Wang, Zhigang Ren, XiaoBo Gu, Ming Yin and Zongze Wu
}

\begin{abstract}
A new efficient root-propagator method based on a uniform linear antenna array is proposed to estimate the directions of arrival (DOAs) and ranges of near-field sources without a priori knowledge of the source number. A non-Hermitian matrix is firstly designed which allows the applications of rooting methods. Then by constructing two propagators, the parameter estimation for near-field sources can be achieved through rooting methods in a decoupled way without eigenvalue decomposition (EVD) and spectrum searches. At last, the importance of the priori source number to source localization problems is studied, and specifically, an improvement is proposed to get rid of this dependence. Numerical examples are carried out to show the effectiveness and validate the theoretical analysis of the proposed root-propagator method. The results show that the proposed method provides more superior performance for near-field source localization than other existing similar methods. Even in the case without a priori knowledge of the source number, the performance of the proposed method is still satisfactory.
\end{abstract}

Index Terms-Root-propagator, near-field, source localization, source number

\section{INTRODUCTION}

For the latest decades, source localization is an important topic to the research of array signal processing, which contributes impressively to civil and military applications [1][3]. In the far-field situation, the wave-front of a source signal is considered plane. The model is only parameterized by the direction-of-arrival (DOA), and plenty of localization methods have been proposed for this situation [4]-[10]. When the sources are located in the Fresnel region, the wavefronts are considered to be spherical. Not only the DOAs, but also the ranges are required to characterize the near-field sources [11]-[20]. The most obvious idea for near-field source localization is extending the classical one-dimensional (1D) DOA estimators to two-dimensional (2D) ones, like the 2D multiple signal classification (MUSIC) method [21]. For many methods, a 2D spectrum search is usually required, which

J. Li, Z. Ren, X. Gu, M. Yin and Z. Wu are with the School of Automation, Guangdong University of Technology, Guangzhou 510000, China (e-mail: jianzhong.li@gdut.edu.cn, renzhigang@gdut.edu.cn, xiaobo.gu@gdut.edu.cn yiming@gdut.edu.cn and zzwu@gdut.edu.cn). J. Li is also with Research Institute of Integrated Circuit Innovation, Guangdong University of Technology, Guangzhou 510000, China. Y. Wang is with Institut d'Electronique et Télécommunications de Rennes (IETR), Université de Nantes, UMR CNRS 6164, Rue Christian Pauc BP 50609, Nantes 44306, France (e-mail: yide.wang@univ-nantes.fr).

This work was supported in part by the National Natural Science Foundation of China under Grants 62001127 and 61673124, and in part by Guangdong Provincial Key-Field Research Program 2018B010115001. (Corresponding authors: Xiaobo Gu, Zongze Wu.) would introduce a high computational burden, limiting its application in some practical scenarios (for example, long-time field work without guaranteed electronic energy due to the fact that algorithms with higher complexities would consume more energy, and therefore lead to an exhaustion more quickly).

The decoupled estimation of the two parameters of nearfield sources is firstly proposed by using high-order cumulant in [22]-[23]. The high number of degrees of freedom provides the possibility to design various matrices containing the information of only one parameter and the two parameters are estimated independently. But they need to be paired through parameter matching algorithms for the source localization. Recently another family of methods is investigated for the localization of near-field sources in a decoupled way [24]-[31]. The 2D spectrum search can be avoided through these methods without additional parameter matching algorithms, bringing a significant improvement to the computational efficiency. These methods consist mainly of two steps. By designing a Hermitian matrix which can eliminate the information of the ranges, the DOAs are firstly estimated. Then the range associated with each estimated DOA is estimated with another Hermitian matrix. [24] estimates the DOA firstly by applying a focusing technique. However, a preestimation technique based on beamforming is necessary. [26]-[27] achieve the DOA estimation with the anti-diagonal elements of the secondorder covariance matrix, but suffer from an aperture loss. The application of high-order cumulant [28]-[31] avoids the aperture loss and achieves better performance due to the high resistance to Gaussian noise. However, all these methods need to construct at least two different Hermitian matrices and usually the eigenvalue decomposition (EVD) has to be applied to each constructed Hermitian matrix. Although in [32] we have proven that it is not necessary to use Hermitian matrices and proposed a new MUSIC-like method with only one EVD and one non-Hermitian matrix, it is applicable only when the constructed matrix is diagonalizable. Besides, the most critical point is that all the existing source localization methods, including near-field and far-field, are developed based on an accurate priori knowledge of the source number. When the number is not known apriori, or cannot be estimated perfectly, the localization through these methods is no longer credible.

Therefore, a new root-propagator-based method is proposed in this paper for near-field source localization using only a single non-Hermitian matrix, where none of the EVD, spectrum search or source number is necessary. Firstly, a non-Hermitian matrix is designed with the fourth-order cumulant to meet the

\section{ACCEPTED MANUSCRIPT}


requirement of the application of rooting methods. Secondly, the way to construct subspaces will be decided according to whether the source number is known or not. Thirdly, two propagators associated with two different parameters are calculated with the corresponding subspaces. Finally, the two localization parameters of near-field sources will be estimated through rooting methods. Compared with existing methods, there are five contributions in the proposed root-propagator method: (i) only one matrix is required for the decoupled estimation of two parameters; (ii) the EVD operation is avoided by calculating propagators; (iii) the rooting method is applied for estimating the parameters without parameter matching steps; (iv) the proposed method is allowed to be carried out with a larger aperture than those used in other methods, leading to a higher estimation accuracy; (v) the effect of the source number is studied and an improvement is proposed to make the proposed method capable of localizing sources without a priori knowledge of the source number.

The rest of this paper is organized as follows. Section II presents the signal model and the problems of near-field source localization. In Section III, the proposed method is described in details, and its complexity is analyzed to show the computational efficiency. Section IV provides several numerical examples to evaluate the performance of the proposed method. At last, this paper is concluded in Section V.

Notation: In this paper, the superscripts $T, H$ and $*$ represent the transpose operation, the conjugate transpose and the complex conjugate respectively. $\operatorname{cum}\{\cdot\}$ stands for the cumulant operation, $E[\cdot]$ is the statistical expectation and $\operatorname{rank}(\cdot)$ denotes the rank of the matrix.

\section{Signal Model and Problem Statement}

In this section, the signal model of near-field source localization will be presented firstly. Then the main problems of localizing near field sources will be discussed.

\section{A. Signal Model}

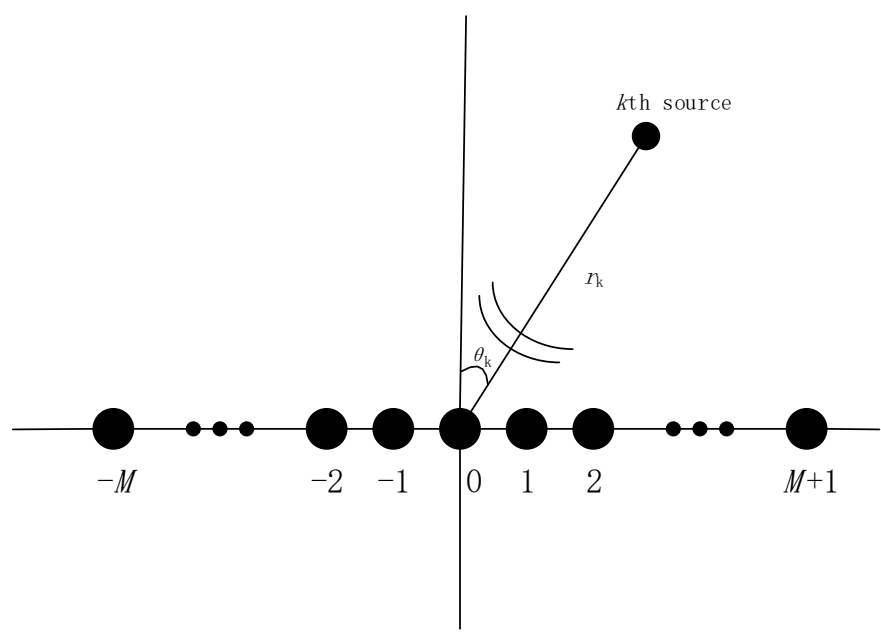

Fig. 1. Near-field source localization with ULA.

As demonstrated in Fig. 1, a uniform linear array (ULA) is adopted in this paper. The array consists of $2 M+2$ antennas, and the inter-antenna spacing is $d$. We take the antenna at the centre as the reference antenna (marked as the 0th antenna). Assume that there are $K(K<2 M+1)$ near-field independent narrow-band signals impinging on the ULA. The output of the $m$ th antenna can be written as

$$
y_{m}(t)=\sum_{k=1}^{K} s_{k}(t) e^{j \varphi_{m k}}+n_{m}(t), t=1,2, \ldots, T,
$$

with $T$ representing the number of snapshots, $s_{k}(t)$ the $k$ th source signal with non-zero kurtosis received at the 0th antenna and $n_{m}(t)$ the additive Gaussian noise (white or coloured) received at the $m$ th antenna. The noises received at different antennas are supposed to be independent from each other and from all the signal sources. $\varphi_{m k}$ is the phase difference due to the propagation time between the $m$ th antenna and 0th one, which can be expressed as

$$
\begin{aligned}
\varphi_{m k} & =\frac{2 \pi}{\lambda}\left(\sqrt{r_{k}^{2}+(m d)^{2}-2 r_{k} m d \sin \theta_{k}}-r_{k}\right) \\
& \approx \omega_{k} m+\phi_{k} m^{2},
\end{aligned}
$$

where

$$
\begin{aligned}
\omega_{k} & =-\frac{2 \pi d}{\lambda} \sin \theta_{k}, \\
\phi_{k} & =\frac{\pi d^{2}}{\lambda r_{k}} \cos ^{2} \theta_{k} .
\end{aligned}
$$

$\lambda$ is the wavelength of the source signal, which satisfies $\lambda \geq 2 d . r_{k}$ and $\theta_{k}$ are the range and DOA of the $k$ th source respectively. For the near-field source localization, the sources are located in the Fresnel region:

$$
r_{k} \in\left(0.62\left(D^{3} / \lambda\right)^{1 / 2}, 2 D^{2} / \lambda\right),
$$

with $D$ being the aperture of the array.

The received signal can be written in the matrix form as follows:

$$
\mathbf{y}(t)=\mathbf{A}(\boldsymbol{\theta}, \mathbf{r}) \mathbf{s}(t)+\mathbf{n}(t),
$$

with $\mathbf{y}(t)$ being the $(2 M+2) \times 1$ received signal vector:

$$
\mathbf{y}(t)=\left[y_{-M}(t), y_{-M+1}(t), \ldots, y_{M+1}(t)\right]^{T},
$$

$\mathbf{s}(t)$ the $K \times 1$ source signal vector:

$$
\mathbf{s}(t)=\left[s_{1}(t), s_{2}(t), \ldots, s_{K}(t)\right]^{T},
$$

$\mathbf{A}(\boldsymbol{\theta}, \mathbf{r})$ the $(2 M+2) \times K$ steering matrix:

$$
\mathbf{A}(\boldsymbol{\theta}, \mathbf{r})=\left[\mathbf{a}\left(\theta_{1}, r_{1}\right), \mathbf{a}\left(\theta_{2}, r_{2}\right), \ldots, \mathbf{a}\left(\theta_{K}, r_{K}\right)\right],
$$

$\mathbf{a}\left(\theta_{k}, r_{k}\right)$ the $(2 M+2) \times 1$ steering vector:

$$
\begin{aligned}
\mathbf{a}\left(\theta_{k}, r_{k}\right)= & {\left[e^{j\left[(-M) \omega_{k}+(-M)^{2} \phi_{k}\right]}, \ldots,\right.} \\
& \left.e^{j\left[(M+1) \omega_{k}+(M+1)^{2} \phi_{k}\right]}\right]^{T},
\end{aligned}
$$

and $\mathbf{n}(t)$ the $(2 M+2) \times 1$ noise vector:

$$
\mathbf{n}(t)=\left[n_{-M}(t), n_{-M+1}(t), \ldots, n_{M+1}(t)\right]^{T} .
$$

\section{B. Problem of Localizing Near-field Sources}

From (2) it is clear that the phase $\varphi_{m k}$ does not change linearly along the elements of the steering vector $\mathbf{a}\left(\theta_{k}, r_{k}\right)$, 
which prevents the application of rooting methods [33]. In near-field source localization, the spectrum search is still necessary for most subspace-based methods, like MUSICbased methods. In addition, the traditional way to obtain the desired subspaces is the application of EVD or singular value decomposition (SVD). The specific group of eigenvectors or singular vectors, corresponding to the $K$ largest eigenvalues or singular values, can span the desired subspaces, but this decomposition is computationally intensive and time-consuming. More importantly, the selection of eigenvectors or singular vectors is directly related to the number of sources $K$, which must be a priori knowledge or an accurate estimate for source localization problems.

Thus, in this paper we investigate a computationally efficient method to localize near-field sources, where the rooting technique can be applied and the EVD operation is not required. Furthermore, the effect of the source number $K$ to source localization is studied, and we propose an improvement for the case when $K$ is unknown or not known precisely.

\section{Proposed Scheme}

In order to show the solution to the above problems step by step, firstly under the assumption that the number of sources $K$ is known, a root-propagator method based on the fourthorder cumulant is proposed to estimate $\phi$ and $\omega$ respectively in Sections A and B. Secondly, the effect of $K$ to the proposed root-propagator method is analyzed and a further improvement is made to get rid of the dependence of $K$ in Section $C$. In Section D, the computational complexity analysis among different methods is provided to make a comparison, revealing the effectiveness of the proposed method.

As described before, the fourth-order cumulant will be adopted in this paper. When the statistic order is greater than 2 , the cumulant of Gaussian noise would be zero [34]:

$$
\operatorname{cum}\left\{n_{p}(t), n_{q}^{*}(t), n_{i}(t), \ldots\right\}=0 .
$$

Consequently, the high-order cumulant shows a superior resistance to the noise following Gaussian distribution. For the simplification of our analysis, the noise term in equations of high-order cumulant can be omitted, and we can focus on the source signal terms.

The fourth-order cumulant of the received signal can be written as [22], [29], [30]:

$$
\begin{aligned}
& \operatorname{cum}\left\{y_{m}(t), y_{n}^{*}(t), y_{p}(t), y_{q}^{*}(t)\right\} \\
& =\sum_{k=1}^{K} c_{4 s_{k}} e^{j\left[(m-n+p-q) \omega_{k}+\left(m^{2}-n^{2}+p^{2}-q^{2}\right) \phi_{k}\right]}, \\
& \quad \operatorname{cum}\left\{s_{m}(t), s_{n}^{*}(t), s_{p}(t), s_{q}^{*}(t)\right\} \\
& \quad=\left\{\begin{array}{cl}
c_{4 s_{k}}, & (m=n=p=q=k) \\
0, & \text { (others })
\end{array}\right.
\end{aligned}
$$

with $c_{4 s_{k}}$ being the fourth-order cumulant of $s_{k}$.

\section{A. $\phi$ Estimation with Priori Source Number}

Firstly, we consider the regular situation where the source number $K$ is known. In this case, we develop a low complexity method to localize near-field sources without EVD and spectrum searches. A matrix of fourth-order cumulant is designed to allow the application of rooting methods. The $(\bar{m}, \bar{n})$ th element of this matrix is given by:

$$
\begin{aligned}
& \mathbf{C}(\bar{m}, \bar{n}) \\
& =\operatorname{cum}\left\{y_{m+1}, y_{m}^{*}, y_{0}, y_{n}^{*}\right\} \\
& =\sum_{k=1}^{K} c_{4 s_{k}} e^{j\left(\omega_{k}+\phi_{k}\right)} e^{j\left(2 m \phi_{k}\right)} e^{-j\left(\omega_{k} n+\phi_{k} n^{2}\right)},
\end{aligned}
$$

with $-M \leq m, n \leq M$ and $\bar{m}=m+M+1, \bar{n}=n+M+1$. This matrix can be expressed as follows:

$$
\mathbf{C}=\mathbf{A}_{1}(\boldsymbol{\phi}) \mathbf{C}_{4 s} \mathbf{A}_{2}^{H}(\boldsymbol{\omega}, \boldsymbol{\phi}) .
$$

$\mathbf{C}_{4 s}$ is a diagonal matrix with the size $K \times K$, and the diagonal entries are $c_{4 s_{1}} e^{j\left(\omega_{1}+\phi_{1}\right)}, c_{4 s_{2}} e^{j\left(\omega_{2}+\phi_{2}\right)}, \ldots, c_{4 s_{K}} e^{j\left(\omega_{K}+\phi_{K}\right)}$ :

$$
\mathbf{C}_{4 s}=\left[\begin{array}{ccc}
c_{4 s_{1}} e^{j\left(\omega_{1}+\phi_{1}\right)} & \ldots & 0 \\
\vdots & & \\
0 & \ldots & c_{4 s_{K}} e^{j\left(\omega_{K}+\phi_{K}\right)}
\end{array}\right]
$$

$\mathbf{A}_{1}(\boldsymbol{\phi})$ and $\mathbf{A}_{2}(\boldsymbol{\omega}, \boldsymbol{\phi})$ are the following two $(2 M+1) \times K$ steering matrices:

$$
\mathbf{A}_{1}(\phi)=\left[\mathbf{a}_{1}\left(\phi_{1}\right), \mathbf{a}_{1}\left(\phi_{2}\right), \ldots, \mathbf{a}_{1}\left(\phi_{K}\right)\right],
$$

$$
\mathbf{A}_{2}(\boldsymbol{\omega}, \boldsymbol{\phi})=\left[\mathbf{a}_{2}\left(\omega_{1}, \phi_{1}\right), \mathbf{a}_{2}\left(\omega_{2}, \phi_{2}\right), \ldots, \mathbf{a}_{2}\left(\omega_{K}, \phi_{K}\right)\right],
$$

with $\mathbf{a}_{1}\left(\phi_{k}\right)$ and $\mathbf{a}_{2}\left(\omega_{k}, \phi_{k}\right)$ being the following two (2M+ 1) $\times 1$ vectors:

$$
\begin{array}{r}
\mathbf{a}_{1}\left(\phi_{k}\right)=\left[e^{j 2(-M) \phi_{k}}, e^{j 2(-M+1) \phi_{k}},\right. \\
\left.\ldots, e^{j 2 M \phi_{k}}\right]^{T}, \\
\mathbf{a}_{2}\left(\omega_{k}, \phi_{k}\right)=\left[e^{j\left[(-M) \omega_{k}+(-M)^{2} \phi_{k}\right]},\right. \\
\left.\ldots, e^{j\left(M \omega_{k}+M^{2} \phi_{k}\right)}\right]^{T} .
\end{array}
$$

We can see from (16) that all the columns of $\mathbf{C}$ can be expressed as a linear combination of the columns of $\mathbf{A}_{1}(\phi)$, and the corresponding expansion coefficients are the products of $\mathbf{C}_{4 s} \mathbf{A}_{2}^{H}(\boldsymbol{\omega}, \boldsymbol{\phi})$. With the assumption that the accurate source number $K$ is known as a priori knowledge, the first $K$ columns of $\mathbf{C}$ can be taken to construct a new matrix $\mathbf{U}_{s 1}$ :

$$
\mathbf{U}_{s 1}=\left[\mathbf{c}_{1}, \mathbf{c}_{2}, \ldots, \mathbf{c}_{K}\right] .
$$

$\mathbf{U}_{s 1}$ spans the same column subspace as $\mathbf{A}_{1}(\phi)$ does. Define

$$
\mathbf{U}_{n 1}=\mathbf{I}_{2 M+1}-\mathbf{U}_{s 1}\left(\mathbf{U}_{s 1}^{H} \mathbf{U}_{s 1}\right)^{-1} \mathbf{U}_{s 1}^{H},
$$

where $\mathbf{I}_{2 M+1}$ is an identity matrix with the size of $(2 M+$ $1) \times(2 M+1)$. It is obvious that

$$
\mathbf{U}_{s 1}^{H} \mathbf{U}_{n 1}=\mathbf{0}_{K \times(2 M+1)},
$$

where $\mathbf{0}_{p \times q}$ is a zero matrix with the size of $p \times q$. Therefore, $\mathbf{U}_{s 1}$ is orthogonal to $\mathbf{U}_{n 1}$, which is equivalent to

$$
\mathbf{A}_{1}^{H}(\phi) \mathbf{U}_{n 1}=\mathbf{0}_{K \times(2 M+1)} .
$$

Comparing 20) with (2), we can see that by using the 
proposed procedure, $2 m \phi_{k}$ is a linear phase that shifts along the antennas of the array. The polynomial for rooting can be expressed as

$$
f_{1}(z)=z^{2 M+1} \mathbf{a}^{T}\left(z^{-1}\right) \mathbf{U}_{n 1} \mathbf{U}_{n 1}^{H} \mathbf{a}(z),
$$

where $\mathbf{a}(z)=\left[z^{-M}, \ldots, z^{-1}, 1, z, \ldots, z^{M}\right]^{T}$ and $z=e^{j 2 \phi}$. Due to the conjugate symmetry of the polynomial $f_{1}(z)$, its roots appear in pairs where one root is the conjugate reciprocal of the other [6]. The roots closest to and inside the unit circle can be used to estimate the parameter $\phi$ :

$$
\hat{\phi}_{k}=\frac{\angle\left(z_{k}\right)}{2},
$$

where $\angle(z)$ is the angle of the complex variable $z$.

\section{B. $\omega$ Estimation with Priori Source Number}

Considering again the cumulant matrix in (16), the rows of $\mathbf{C}$ can be considered as a linear combination of those of $\mathbf{A}_{2}^{H}(\boldsymbol{\omega}, \phi)$. We can construct another matrix as follows:

$$
\mathbf{U}_{s 2}=\left[\mathbf{r}_{1}, \mathbf{r}_{2}, \ldots, \mathbf{r}_{K}\right],
$$

where $\mathbf{r}_{1}^{H}, \mathbf{r}_{2}^{H}, \ldots, \mathbf{r}_{K}^{H}$ denote to the first $K$ rows of $\mathbf{C}$. Define

$$
\mathbf{U}_{n 2}=\mathbf{I}_{2 M+1}-\mathbf{U}_{s 2}\left(\mathbf{U}_{s 2}^{H} \mathbf{U}_{s 2}\right)^{-1} \mathbf{U}_{s 2}^{H},
$$

Similar to the previous discussion, $\mathbf{A}_{2}(\boldsymbol{\omega}, \phi)$ is orthogonal to $\mathbf{U}_{n 2}$ :

$$
\mathbf{A}_{2}^{H}(\boldsymbol{\omega}, \phi) \mathbf{U}_{n 2}=\mathbf{0}_{K \times(2 M+1)} .
$$

The phase of the $k$ th steering vector $\mathbf{a}_{2}\left(\omega_{k}, \phi_{k}\right)$ is $m \omega_{k}+$ $m^{2} \phi_{k}$, which of course does not change linearly along the elements of the steering vector and rooting methods cannot be applied directly. However, $m \omega_{k}$ alone does shift linearly. As we have already estimated $\phi_{k}$, the $k$ th steering vector $\mathbf{a}_{2}\left(\omega_{k}, \hat{\phi}_{k}\right)$ can be decomposed as

$$
\mathbf{a}_{2}\left(\omega_{k}, \hat{\phi}_{k}\right)=\boldsymbol{\Gamma}\left(\hat{\phi}_{k}\right) \mathbf{a}_{21}\left(\omega_{k}\right),
$$

with

$$
\boldsymbol{\Gamma}\left(\hat{\phi}_{k}\right)=\left[\begin{array}{cccc}
e^{j(-M)^{2} \hat{\phi}_{k}} & 0 & \ldots & 0 \\
0 & e^{j(-M+1)^{2} \hat{\phi}_{k}} & \ldots & 0 \\
\vdots & & & \\
0 & 0 & \ldots & e^{j M^{2} \hat{\phi}_{k}}
\end{array}\right]
$$

and

$$
\mathbf{a}_{21}\left(\omega_{k}\right)=\left[e^{j(-M) \omega_{k}}, \ldots, e^{j M \omega_{k}}\right]^{T} .
$$

According to the above discussion, we have $\mathbf{A}_{2}(\boldsymbol{\omega}, \phi) \perp \mathbf{U}_{n 2}$. Substitute each estimated $\hat{\phi}_{k}$ into 32 and a new matrix $\mathbf{V}_{n k}$ orthogonal with $\mathbf{a}_{21}\left(\omega_{k}\right)$ can be formed as

$$
\mathbf{V}_{n k}=\Gamma^{H}\left(\hat{\phi}_{k}\right) \mathbf{U}_{n 2} .
$$

From (33), we observe that the phase changes linearly along the elements of $\mathbf{a}_{21}\left(\omega_{k}\right)$, satisfying the requirement for applying rooting methods [33]. Therefore, another polynomial could be expressed as follows:

$$
f_{2}(z)=z^{2 M+1} \mathbf{a}^{T}\left(z^{-1}\right) \mathbf{V}_{n k} \mathbf{V}_{n k}^{H} \mathbf{a}(z),
$$

where $z=e^{j \omega}$. Unlike the estimation of $\phi$, there is only one pair of roots that is on the unit circle, which represents the $\omega_{k}$ estimate for the substituted $k$ th $\phi$ estimate $\hat{\phi}_{k}$. Therefore, the paring of $\omega$ and $\phi$ is achieved automatically. No extra pairing algorithms are required, reducing the complexity of the algorithm. Take the root closest to and inside the unit circle and we can estimate $\omega_{k}$ by

$$
\hat{\omega}_{k}=\angle(\hat{z}) \text {. }
$$

Substituting $\hat{\phi}_{k}$ and $\hat{\omega}_{k}$ into $(3)$ and (4), the DOA and range estimates of the $k$ th near-field source can be achieved.

Remark A: Other methods for decoupled near-field source localization, such as HOS in [29], SOS in [26] and LOFNS in [27], work well under the requirement of $d \leq \frac{\lambda}{4}$. In those methods, the $k$ th DOA is estimated through a steering vector with the $m$ th element being $e^{j 2 m \omega}$. In order to guarantee the uniqueness of DOA estimates, the requirement that $2 \omega=-\frac{4 \pi d}{\lambda} \sin \theta_{k} \in[-\pi, \pi]$ must be satisfied. As the result, we have $d \leq \frac{\lambda}{4}$, limiting the array aperture to only $\frac{\lambda M}{2}$. For the proposed method, the DOA estimation is carried out with the steering vector whose $m$ th phase is $e^{j m \omega}$ as shown in 33 . In this case, it only requires $d \leq \frac{\lambda}{2}$. Therefore, our method is also effective for a ULA with a larger value of $d$. The corresponding array aperture is $\lambda M$, which leads to a smaller angular resolution cell and, hence, potentially a higher estimation accuracy.

\section{Improvement without Priori Source Number}

Source number detection and source localization are two major applications of antenna arrays [35]. For source localization problems, one of the basic assumptions is that the number of sources $K$ is known. Nearly all the source localization methods rely heavily on the priori source number (for example, in ESPRIT-based methods, $K$ eigenvectors are used to form the signal subspace). When the source number $K$ cannot be known or is not estimated accurately, the source localization procedure cannot be carried out properly.

For the proposed root-propagator method in Sections A and $\mathrm{B}$, the source number $K$ is used to decide the selection of vectors to form the matrices $\mathbf{U}_{s 1}$ and $\mathbf{U}_{s 2}$ (see (22) and (28)). Assume that the number of sources $K$ is no longer known, and $\tilde{K}$ columns of $\mathbf{C}$ are taken to form $\mathbf{U}_{s 1}$. When $\tilde{K}<K$, we have $\operatorname{rank}\left(\mathbf{U}_{s 1}\right) \leq \tilde{K}<K=\operatorname{rank}\left(\mathbf{A}_{1}(\phi)\right)$. The orthogonality between $\mathbf{A}_{1}(\phi)$ and $\mathbf{U}_{n 1}$ cannot hold true, resulting in the failure of the estimation of $\phi$. When $\tilde{K}>K$, the size of $\mathbf{U}_{s 1}$ is $(2 M+1) \times \tilde{K}$ and $\mathbf{U}_{s 1}$ is not full columnrank. Notice that in 23), there is a term for inverse operation: $\left(\mathbf{U}_{s 1}^{H} \mathbf{U}_{s 1}\right)^{-1}$, which means that $\mathbf{U}_{n 1}$ cannot be constructed when $\tilde{K}>K$. This analysis also holds true for $\mathbf{U}_{s 2}$ and $\mathbf{U}_{n 2}$.

In order to obtain $\mathbf{U}_{n 1}$ and $\mathbf{U}_{n 2}$, it is necessary to make the items $\mathbf{U}_{s 1}^{H} \mathbf{U}_{s 1}$ and $\mathbf{U}_{s 2}^{H} \mathbf{U}_{s 2}$ always full rank before the inverse operation. Firstly, $\tilde{K}$ columns and rows will be taken to form $\mathbf{U}_{s 1}$ and $\mathbf{U}_{s 2}$. (22) and (28) are replaced with

$$
\mathbf{U}_{s 1}=\left[\mathbf{c}_{1}, \mathbf{c}_{2}, \ldots, \mathbf{c}_{\tilde{K}}\right]
$$

and

$$
\mathbf{U}_{s 2}=\left[\mathbf{r}_{1}, \mathbf{r}_{2}, \ldots, \mathbf{r}_{\tilde{K}}\right]
$$




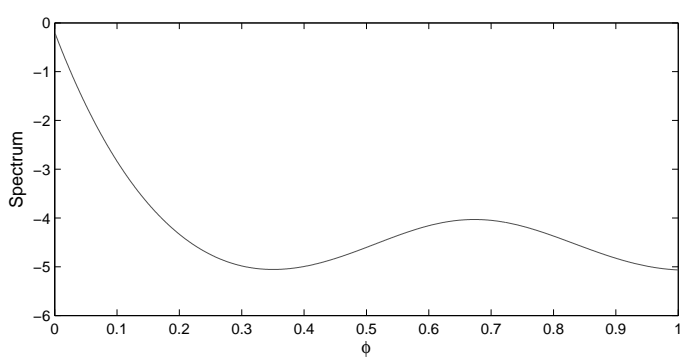

(a) Spectrum when $\mu=0$

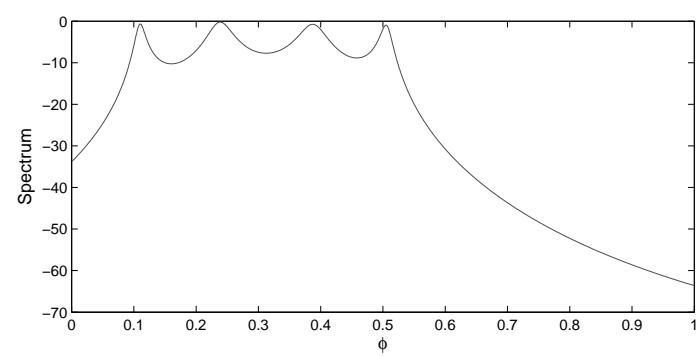

(b) Spectrum when $\mu=10^{-5}$

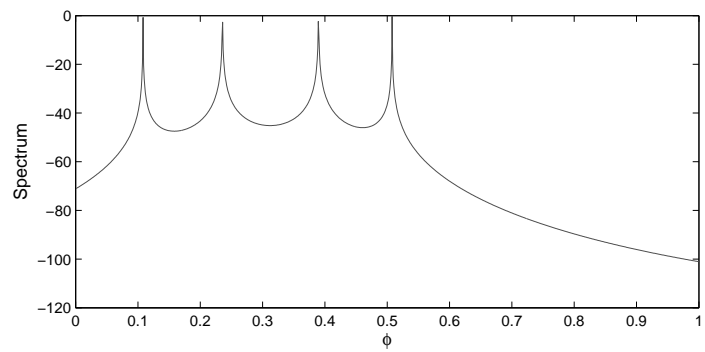

(c) Spectrum when $\mu=10^{-7}$

Fig. 2. Spectra of $\phi$ with 4 sources when $\tilde{K}=2 M+1$ and $M=2$.

Then define

$$
\mathbf{U}_{n 1}=\mathbf{I}_{2 M+1}-\mathbf{U}_{s 1}\left(\mathbf{U}_{s 1}^{H} \mathbf{U}_{s 1}+\mu \mathbf{I}_{\tilde{K}}\right)^{-1} \mathbf{U}_{s 1}^{H},
$$

and

$$
\mathbf{U}_{n 2}=\mathbf{I}_{2 M+1}-\mathbf{U}_{s 2}\left(\mathbf{U}_{s 2}^{H} \mathbf{U}_{s 2}+\mu \mathbf{I}_{\tilde{K}}\right)^{-1} \mathbf{U}_{s 2}^{H} .
$$

According to matrix theory, $\mathbf{U}_{s 1}^{H} \mathbf{U}_{s 1}$ and $\mathbf{U}_{s 2}^{H} \mathbf{U}_{s 2}$ are positive semi-definite. There exist uncountable positive values for $\mu$ $(\mu>0)$ to make $\mathbf{U}_{s 1}^{H} \mathbf{U}_{s 1}+\mu \mathbf{I}_{\tilde{K}}$ and $\mathbf{U}_{s 2}^{H} \mathbf{U}_{s 2}+\mu \mathbf{I}_{\tilde{K}}$ always full rank. When $\mu$ is small enough, we have $\mathbf{U}_{s 1}^{H} \mathbf{U}_{s 1}+\mu \mathbf{I}_{\tilde{K}} \approx$ $\mathbf{U}_{s 1}^{H} \mathbf{U}_{s 1}$ and $\mathbf{U}_{s 2}^{H} \mathbf{U}_{s 2}+\mu \mathbf{I}_{\tilde{K}} \approx \mathbf{U}_{s 2}^{H} \mathbf{U}_{s 2}$. The impact of the additive identity matrix to the orthogonality between the steering matrices and the orthogonal matrices can be negligible.

Fig. 2 shows three different spectra with four near-field sources when $2 M+1$ columns are taken to form $\mathbf{U}_{s 1}$ (i.e., $\tilde{K}=2 M+1$ ). In (a), $\mu=0$ is considered for the case where no processing is made to $\mathbf{U}_{s 1}^{H} \mathbf{U}_{s 1}$. The term is not full rank and the corresponding spectrum is actually a random one. The impact of different values for $\mu$ to the performance of the proposed method is shown in (b) and (c). When $\mu=10^{-5}$, we can see that the peaks are not sharp enough, which may result in some bias for the estimates. When $\mu=10^{-7}$, the

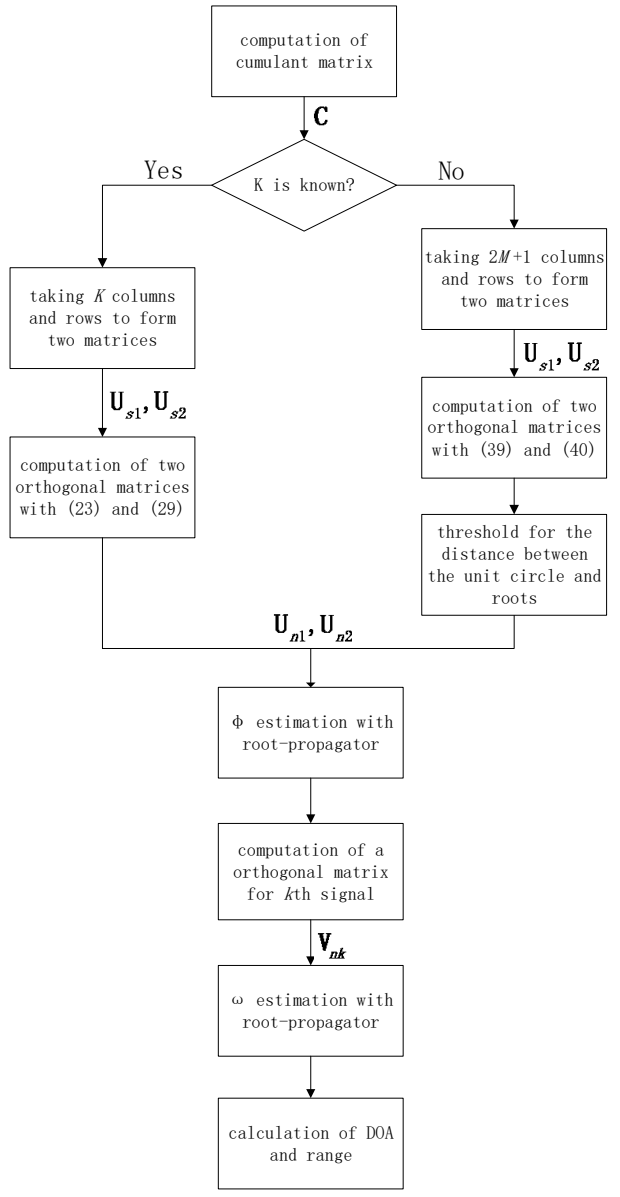

Fig. 3. Procedure of the proposed root-propagator method.

peaks are well distinguishable and each peak may refer to the parameter value in the ground truth.

It is worthy to note that, although the introduction of $\mu \mathbf{I}_{\tilde{K}}$ can help to get rid of the dependence of the priori knowledge of the source number, the selection of $\mu$ must be comprehensively considered in practice. When $\mu$ is not small enough, this processing may affects the estimation similarly to the noise. Both the estimation accuracy and the resolution ability may be poor. When $\mu$ is too small, the term $\mu \mathbf{I}_{\tilde{K}}$ may not work, and the propagators constructed with 39 p and (40) would fail. Therefore, this hyperparameter selection must be comprehensively considered according to the practical environment, including signal-to-noise radio (SNR) and the numbers of antennas and sources. Through simulations, we find that generally $\mu=10^{-7}$ can well improve the estimation when the SNR is high. If the SNR is not high enough and there are much more antennas than sources, the value of $10^{-3}$ for $\mu$ would be more suitable.

Remark B: Theoretically, the proposed method can work when $\tilde{K} \geq K$. In order to guarantee that $\mathbf{U}_{n 1}$ and $\mathbf{U}_{n 2}$ are orthogonal with all the steering vectors, we suggest in practice to take $\tilde{K}=2 M+1$ columns or rows to form $\mathbf{U}_{s 1}$ or $\mathbf{U}_{s 2}$, even though the corresponding computational complexity will 
be a little higher.

Remark $C$ : In the proposed method, a cumulant matrix $\mathbf{C}$ with the size $(2 M+1) \times(2 M+1)$ is designed, which is used for estimating both $\omega$ and $\phi$. Therefore, with the proposed method $2 M$ sources can be located at most, which can be verified by the spectra in Fig. 2 .

\section{Complexity Analysis}

As for the computational complexity, we select flops to evaluate the method. The definition of one flop is a floatingpoint addition or multiplication operation. The procedure of the proposed root-propagator method is shown in Fig. 3. In the case where the number of sources is available, the implementation of the proposed root-propagator method and the corresponding computational complexity can be summarized as follows:

1) Estimate $\mathbf{C}$ from $\mathbf{y}(\mathrm{t})$ with 15 .

$$
9(2 M+1)^{2} T \text { flops }
$$

2) Take $K$ columns and rows to form $\mathbf{U}_{s 1}$ and $\mathbf{U}_{s 2}$ with (22) and (28).

$$
2(2 M+1) K \text { flops }
$$

3) Estimate $\mathbf{U}_{n 1}$ and $\mathbf{U}_{n 2}$ from $\mathbf{U}_{s 1}$ and $\mathbf{U}_{s 2}$ with 23) and 29.

$$
2(2 M+1)^{2} K \text { flops }
$$

4) Estimate $\phi$ with the root-propagator method 26.

$$
(2 M+1) K^{2} \text { flops }
$$

5) Estimate $\mathbf{V}_{n k}$ and $\omega_{k}$ with (34) and (35).

$$
(2 M+1)^{3}+(2 M+1) K^{2} \text { flops }
$$

6) Estimate the DOA $\theta_{k}$ and range $r_{k}$ from the estimated $\hat{\phi}_{k}$ from $\hat{\omega}_{k}$ with 3 and (4).

$9 K$ flops

In conclusion, the computational complexity of the proposed root-propagator method is about in the order of $O((2 M+$ $\left.1)^{2} T+(2 M+1)^{3}+(2 M+1)^{2} K+(2 M+1) K^{2}\right)$. The implementation of HOS [29], MOS [30] and SOS [26] includes the construction of two matrices, the application of EVD to each matrix and the spectrum searches. The complexity is roughly in the order of $O\left((2 M+1)^{2} T+(2 M+1)^{3}+(2 M+1) K^{2}+\right.$ $\left.N(2 M+1)^{2}\right)$ with $N$ being the grid number for spectrum searches $(N \gg M, K)$. Besides the computational complexity, constructing two different Hermitian matrices and applying the EVD to each of the two Hermitian matrices also makes the implementations of the methods much more lengthy than that of the proposed root-propagator method.

Remark D: When the source number is not known, the proposed method just needs to replace $K$ with $\tilde{K}$, process $\mathbf{U}_{s 1}^{H} \mathbf{U}_{s 1}$ and $\mathbf{U}_{s 2}^{H} \mathbf{U}_{s 2}$ with an identity matrix, and replace (22), 28), 23) and 29) with (37), 38, 39) and (40). The corresponding complexities of Steps 2) and 3) would be replaced by $2(2 M+1) \tilde{K}$ and $2(2 M+1)^{2} \tilde{K}$ flops. In this case, it is also necessary to set a threshold for the distance between the unit circle and roots, which allows the determination of the true roots. And the corresponding complexity can be neglected. In conclusion, the extra computational complexities are very small compared with other parts. The complexity when $K$ is unknown is only a little higher than that when $K$ is known as a priori knowledge. But for other methods, the estimator of the source number, such as the Akaike information criterion (AIC), Rissanen's minimum description length (MDL) principle [36] and Bayesian information criterion (BIC) [37], must be applied first. The corresponding complexity should also be considered along with those of the localization methods.

Remark E: Theoretically, the fourth-order cumulant can eliminate the effect of Gaussian noise, and achieve unbiased estimation for parameters $\phi$ and $\omega$. However in practical applications, the number of snapshots received by the array is always finite. The fourth-order cumulant of the noise would not be zero for a limited number of snapshots. The performance of the proposed method would still be affected by the noise variance, but not as much as the methods based on the second-order statistics. Indeed, the mean square error (MSE) of the estimation is roughly in inverse proportion to the number of snapshots. In most cases where there are less than 10 antennas, most methods can estimate DOA with an MSE smaller than $10^{-1}$ degree when more than $10^{5}$ snapshots are received. For range estimation, the MSE can be as low as $10^{-1.5}$ wavelength.

\section{NUMERICAL EXAMPLES}

In this section, several numerical examples are displayed to evaluate the performance of the proposed root-propagator method. The results will be compared with some existing similar methods such as HOS in [29], SOS in [26], LOFNS in [27] and the joint-diagonalization method (JDM) in [12]. An array of 8 antennas is applied in all the examples (i.e., $M=3$ ). For the proposed root-propagator method, the maximum interelement spacing of the array $d$ can be $\frac{\lambda}{2}$. For other methods, $d$ has to be no larger than $\frac{\lambda}{4}$ due to their limitation. Examples 1 and 2 are carried out with the priori knowledge of the source number while Example 3 is mainly to demonstrate the performance when the source number is not known. Examples 4 and 5 are designed to verify the ability of source number estimation and source detection.

Example 1-Performance versus SNR: For the following examples, the root mean square error (RMSE) of estimates is used to evaluate the performance of different methods, which is defined as follows:

$$
R M S E=\sqrt{\frac{\sum_{p=1}^{P}\left|\hat{\alpha}_{p}-\alpha_{\text {true }}\right|^{2}}{P}},
$$

where $\hat{\alpha}_{p}$ is the estimate in the $p$ th trial, $\alpha_{\text {true }}$ stands for the true value and $P$ represents the number of independent Monte Carlo trials. The definition of SNR is given as follows:

$$
S N R=10 \log _{10} \frac{\sum_{k=1}^{K} P_{s_{k}}}{\sigma^{2}},
$$

where $P_{s_{k}}$ represents the power of the $k$ th signal. Two wellseparated near-field sources are considered in the examples, 


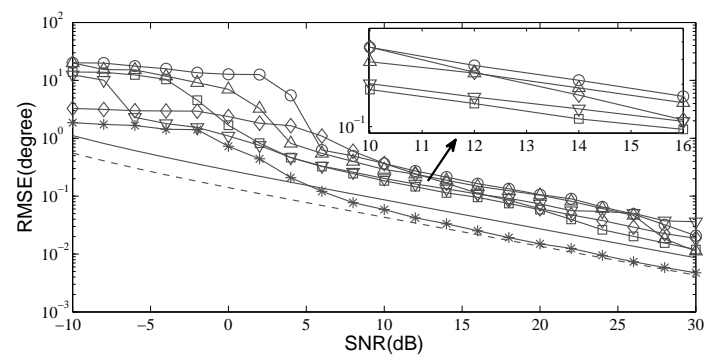

(a) DOA RMSEs versus SNR (1st Source)

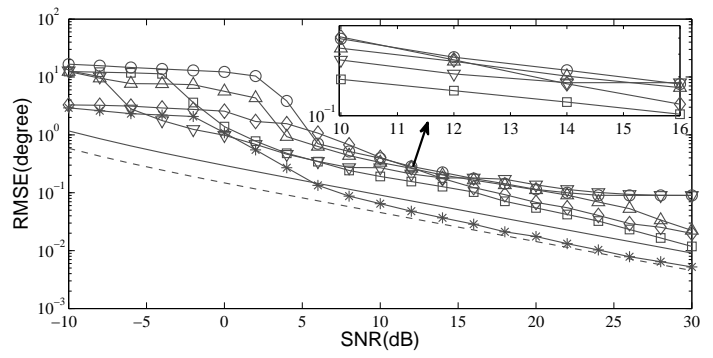

(b) DOA RMSEs versus SNR (2nd Source)

Fig. 4. RMSEs of the DOA estimates for the near-field sources $(\diamond$ : proposed root-propagator with $d=\frac{\lambda}{4} ; \nabla$ : LOFNS; $\bigcirc$ : SOS; $\square$ : HOS; $\triangle$ : JDM; -: $\mathrm{CRB} ; *$ : proposed root-propagator with $d=\frac{\lambda}{2} ;--$ : CRB with $d=\frac{\lambda}{2}$ ).

located at $\left(-8^{\circ}, 1.3 \lambda\right)$ and $\left(20^{\circ}, 2.6 \lambda\right)$ respectively. Assume that the number of snapshots is 200 , and the SNR varies from $-10 \mathrm{~dB}$ to $30 \mathrm{~dB}$. In order to offer a clear comparison, the proposed method is firstly run with $d=\frac{\lambda}{4}$ to meet the same simulation environment with other methods. The corresponding Cramer-Rao Bound (CRB) given in [16] is also provided. Specifically, the proposed method can be carried out with $d=\frac{\lambda}{2}$, and the corresponding RMSEs are added in the figures to make a comparison among the best performance that each method can achieve. All the results plotted in Figs. 4 and 5 are obtained through 1000 independent trials.

Comparing HOS with SOS, the excellent resistance of high-order cumulant to Gaussian noise shows a promotion of the estimation accuracy. Indeed, for most second-order statistics methods (like SOS and LOFNS), the independent DOA estimation often suffers from an aperture loss, where the effective antennas are fewer than the practical ones (the dimension of the covariance matrix for DOA estimation is only $(M+1) \times(M+1)$ while the array consists of $2 M+2$ antennas). As the result, the capacity of the system (i.e., the maximum number of sources that the system can estimate with the array) is reduced and the accuracy is degraded. For the range estimation, these second-order methods do not suffer from the aperture loss, but it relies on the DOA estimation, and the estimation accuracy is also impacted indirectly. The negative effect would be very distinct when the numbers of sources and array antennas are close. Therefore, the secondorder statistics methods often require that the number of array antennas should be much bigger than that of the sources to relieve the imperfection: $2 M+1 \gg K$. For other secondorder statistics methods (like JDM which provides a similar performance with SOS and LOFNS), no aperture loss needs

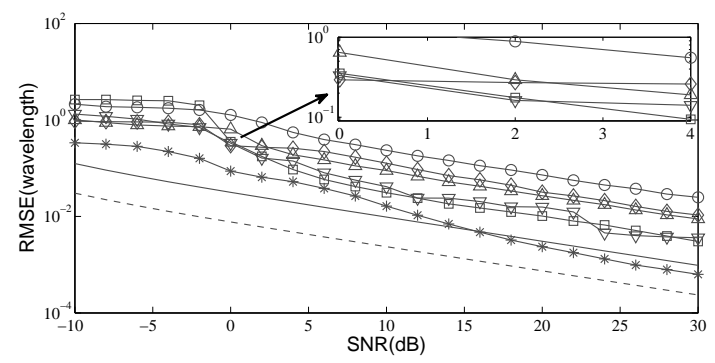

(a) Range RMSEs versus SNR (1st Source)

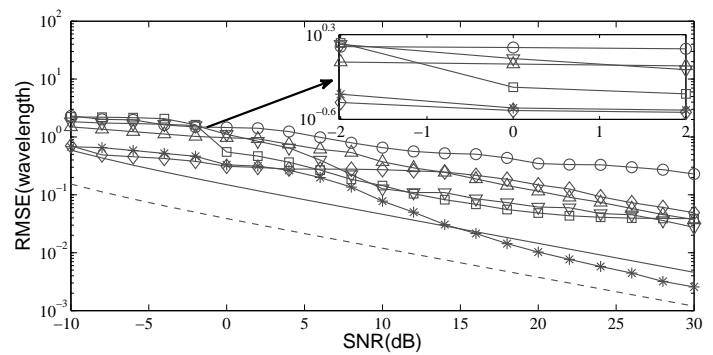

(b) Range RMSEs versus SNR (2nd Source)

Fig. 5. RMSEs of the range estimates for the near-field sources $(\diamond$ : proposed root-propagator with $d=\frac{\lambda}{4} ; \nabla$ : LOFNS; $\bigcirc$ : SOS; $\square$ : HOS; $\triangle$ : JDM; - : $\mathrm{CRB}$; *: proposed root-propagator with $d=\frac{\lambda}{2}$; -- : CRB with $d=\frac{\lambda}{2}$ ).

to be considered. However, the effect of the noise cannot be ignored. The dependence on optimization techniques also affects the performance of JDM, which may produce local optimal solutions instead of global optimal ones. Besides, all the existing methods of this family need to limit the interelement spacing of the array $d \leq \frac{\lambda}{4}$, which also results in a smaller aperture. Conversely, the root-propagator method enables the estimation to be carried out with a larger value of $d$ (i.e., a larger aperture). When $d=\frac{\lambda}{4}$, the proposed method performs almost the same as the other methods. However, when $d=\frac{\lambda}{2}$, the proposed root-propagator performs much better than the other methods, especially for the DOA estimation. The RMSEs are lower than those of all the other methods, as well as the CRBs with $d=\frac{\lambda}{4}$. By comparing the performance of different sources, it can be seen that the accuracies of the DOA estimates are almost the same. However, for the range estimation the RMSEs can tell an apparent difference. The estimation accuracy will be improved if the sources are located closer to the antenna array, which has also been stated in the theoretical analysis in [38].

Example 2-Performance versus Number of Snapshots: In the second example, the relationship between the RMSE and the number of snapshots is studied. The simulation parameters are almost the same with those in Example 1, except the SNR and the number of snapshots. Let the number of snapshots vary from 10 to 10000 . The results with the SNR being $15 \mathrm{~dB}$ are shown in Figs. 6 and 7.

In Fig. 6, when there are less than 1000 snapshots received at the array, HOS outperforms SOS, LOFNS and JDM for DOA estimation benefiting from the high resistance to Gaussian noise. However, HOS reaches its upper limit of estimation accuracy when the number of snapshots is more 


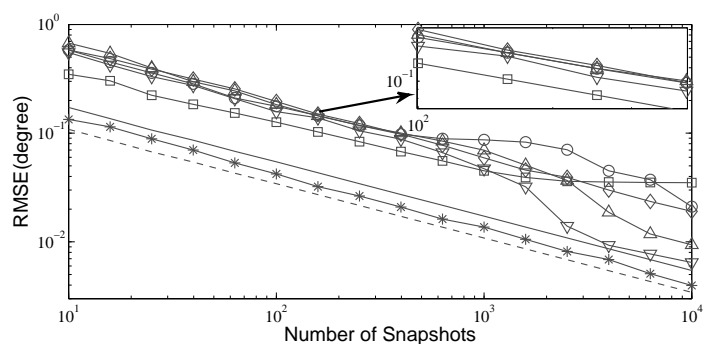

(a) DOA RMSEs versus Snapshots (1st Source)

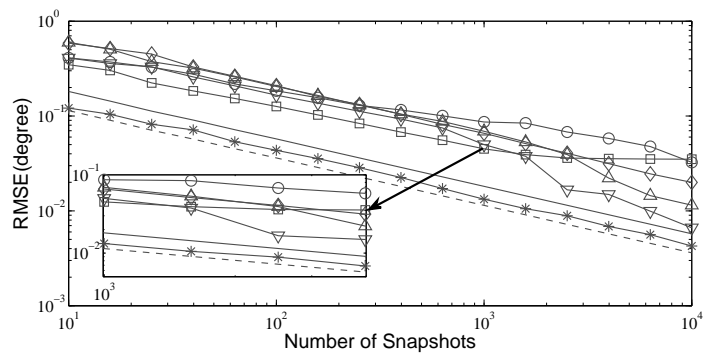

(b) DOA RMSEs versus Snapshots (2nd Source)

Fig. 6. RMSEs of the DOA estimates for the near-field sources $(\diamond:$ proposed root-propagator with $d=\frac{\lambda}{4} ; \nabla$ : LOFNS; $\bigcirc$ : SOS; $\square$ : HOS; $\triangle$ : JDM; -: $\mathrm{CRB}$; $*$ : proposed root-propagator with $d=\frac{\lambda}{2} ;--$ : CRB with $d=\frac{\lambda}{2}$ ).

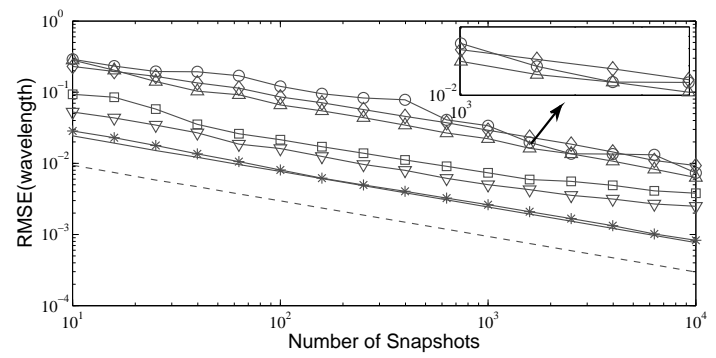

(a) Range RMSEs versus Snapshots (1st Source)

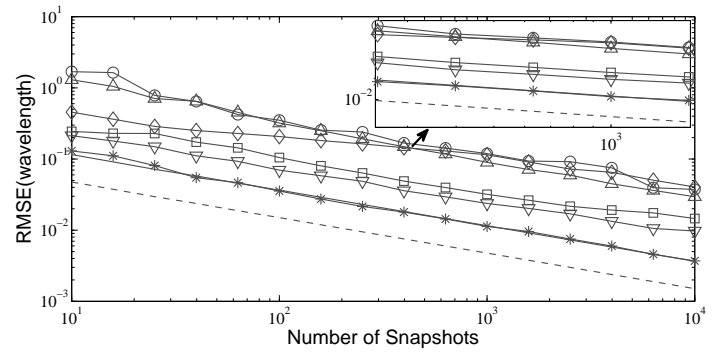

(b) Range RMSEs versus Snapshots (2nd Source)

Fig. 7. RMSEs of the range estimates for the near-field sources $(\diamond$ : proposed root-propagator with $d=\frac{\lambda}{4} ; \nabla$ : LOFNS; $\bigcirc$ : SOS; $\square$ : HOS; $\triangle$ : JDM; -: $\mathrm{CRB}$; $*$ : proposed root-propagator with $d=\frac{\lambda}{2} ;--$ : CRB with $d=\frac{\lambda}{2}$ ).

than 1000. LOFNS would provide a better estimation even though it is based on second-order covariance matrix. As for the proposed root-propagator method, when $d=\frac{\lambda}{4}$ it does not perform better than the other methods. But when $d=\frac{\lambda}{2}$, the estimation accuracy shows an impressive improvement with the increasing of snapshots. It performs much better than the other methods for all the numbers of snapshots, revealing its

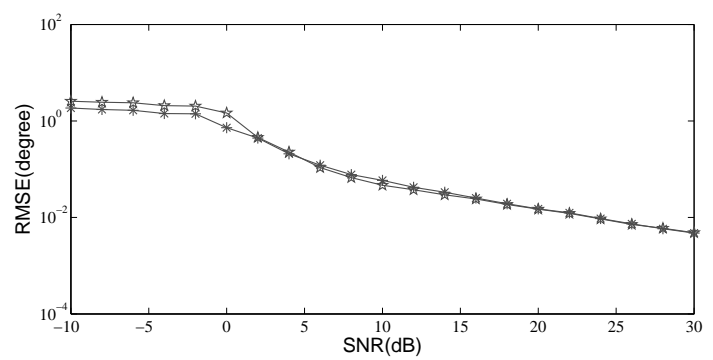

(a) DOA RMSEs versus SNR (1st Source)

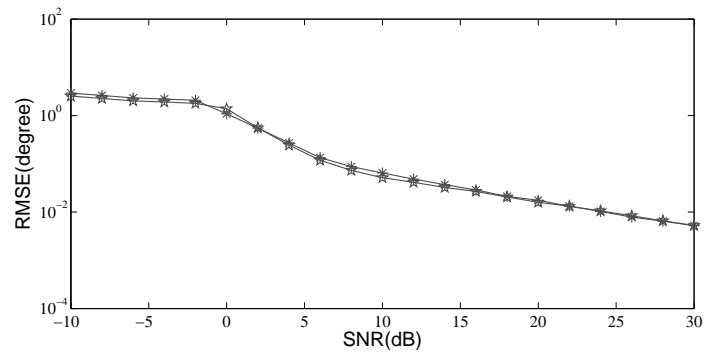

(b) DOA RMSEs versus SNR (2nd Source)

Fig. 8. RMSEs of the DOA estimates for the two near-field sources $(*$ : proposed root-propagator with $K$ known; th: proposed root-propagator with $K$ unknown).

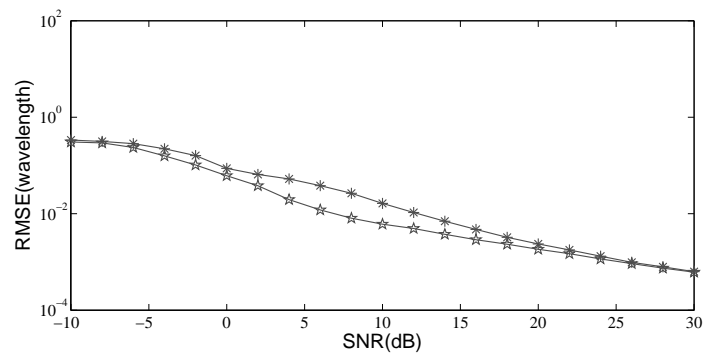

(a) Range RMSEs versus SNR (1st Source)

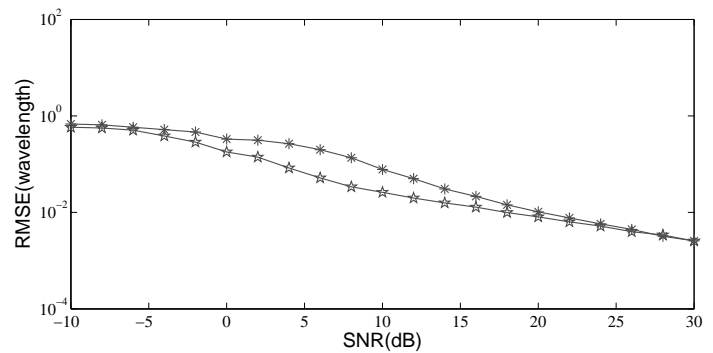

(b) Range RMSEs versus SNR (2nd Source)

Fig. 9. RMSEs of the range estimates for the two near-field sources $(*$ : proposed root-propagator with $K$ known; th: proposed root-propagator with $K$ unknown).

robustness. The range estimation is very sensitive to the noise. Although LOFNS, SOS and JDM are all based on the secondorder statistics, LOFNS provides the best performance, which benefits from the fact that the effect of noise is eliminated in LOFNS. High-order methods can still show some resistance to the noise even though the number of snapshots is small. But the effect is not as good as the technique used in LOFNS.

Example 3-Performance without Priori Source Number: The

\section{ACCEPTED MANUSCRIPT}




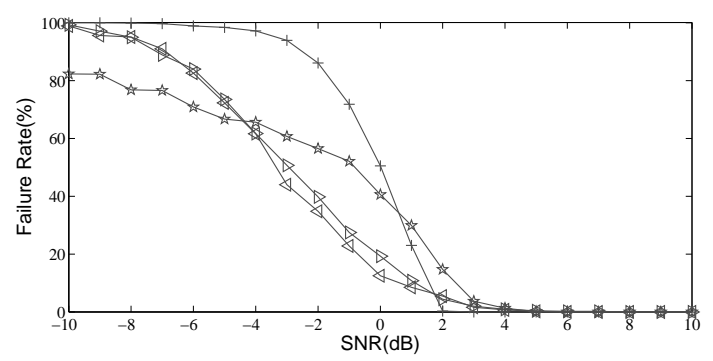

Fig. 10. Failure rate of source number estimation versus SNR ( MDL; +: BIC; 放: proposed root-propagator with $K$ unknown).

third example, of which the simulation conditions are the same with those in Example 1, would investigate the performance in the situation where the number of sources $K$ is not known. The effectiveness of the proposed root-propagator method is already shown through some spectra in Fig. 2, and the RMSEs versus the SNR are displayed in Figs. 8 and 9. In order to provide intuitive results, the RMSEs of the proposed method when $K$ is known are also plotted as a comparison.

It can be seen from the figures that the proposed rootpropagator method performs well even without the priori source number. For the two different situations (knowing the source number and the contrary respectively), the rootpropagator method shows only a small difference, especially for the DOA estimation. When the SNR is not high enough, the proposed root-propagator method without $K$ performs even better. The robustness and accuracy of the root-propagator method are enhanced by using all the $2 M+1$ columns and rows of the cumulant matrix, although the computational complexity would be higher than that when $K$ is known. When the SNR gets higher, the estimation accuracies achieved by the root-propagator in the two situations are almost the same while the other existing methods are unable to work at all without source number estimation. Compared with the RMSEs in Example 1, the root-propagator provides a much better performance than the other methods, in terms of both the computational complexity and estimation efficiency, even though the other methods are carried out with the priori source number.

Example 4-Failure Rate of Source Number Estimation versus SNR: The fourth example is carried out to verify the failure rate of the estimation of the source number $K$, where the simulation conditions are the same with those in Example 1. Indeed, the estimation of $K$ is achieved implicitly when estimating $\phi$. A hyperparameter is set to determine whether a root can be considered as the desired one. Here, the hyperparameter is a threshold for the distance between the unit circle and roots, which is set to be 0.1 . When the distance is smaller than the threshold, the corresponding root can be used to estimate $\phi$. The number of these roots is the estimation of the source number, and the failure rate of this estimation versus SNR is shown in Fig. 10.

From the figure, we can see that the estimation is very effective. When the SNR is $0 \mathrm{~dB}$, the failure rate is about $40 \%$. As the SNR grows, the failure rate drops significantly. The source number can be perfectly estimated even when

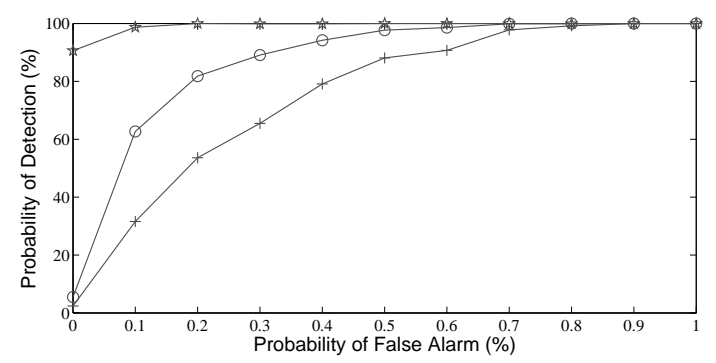

Fig. 11. Probability of Detection versus Probability of False Alarm (- - : $\mathrm{SNR}=0 \mathrm{~dB} ;--$ 支: $\mathrm{SNR}=-3 \mathrm{~dB} ;-$. 文: $\mathrm{SNR}=-5 \mathrm{~dB}$ ).

the SNR is about only $4 \mathrm{~dB}$. Compared with other methods specifically for source number estimation (AIC, MDL and BIC), the proposed method shows a similar result. We can also see that when the SNR is extremely low, the proposed method outperforms others, revealing the better resistance to the noise.

Example 5-Probability of Detection versus Probability of False Alarm: The relationship between the probabilities of detection and false alarm is studied in the fifth example to verify the receive-operator characteristics (ROC) of the proposed method. Let $N_{d}$ denote the number of the trials in which at least one source is detected with the proposed method. When source signal exists in the situation, the ratio between $N_{d}$ and the number of total trials refers to the probability of detection. But in the situation where no source exists, this ratio refers to the probability of false alarm.

According to the constant false alarm rate (CFAR) definition, two different situations are designed in this example. Firstly, the situation is considered where there is only noise received by the array. By changing the threshold which stands for the distance between the unit circle and roots, we can obtain the corresponding probabilities of false alarm. Secondly, we consider another situation where one source located at $\left(-8^{\circ}, 1.3 \lambda\right)$ exists as well as the noise. The probability of false alarm varies from 0 to 1 , and the corresponding threshold is used to obtain the probability of detection. In this example, 200 snapshots are received and all the probabilities are decided with 10000 trials. Fig. 11 shows the results of the proposed method when the SNRs are $-5,-3$ and $0 \mathrm{~dB}$ respectively. We can see that as the SNR increases, the probability of detection shows a significant improvement. When the SNR is $0 \mathrm{~dB}$, the proposed method can detect the source with the probability of nearly $100 \%$ even though the probability of false alarm is only $10 \%$.

\section{CONCLUSION}

In this paper, a root-propagator method is proposed to localize near-field sources. For the proposed method, an array with a larger aperture can be adopted to improve the localization accuracy. Only one matrix needs to be constructed and the computationally burdensome EVD and the spectrum search are avoided efficiently, leading to a much lower computational complexity than those of other methods. Specifically, the case without the priori source number is studied and an improvement is proposed to deal with this problem. The effectiveness

\section{ACCEPTED MANUSCRIPT}


and performance of the proposed method are verified with several numerical examples.

\section{REFERENCES}

[1] F. Soldovier, L. L. Monte, and D. Erricolo, "Tunnel detection and localisation via multi-monostatic radio frequency tomography using magnetic sources,' IET Radar, Sonar Navigation, vol. 6, no. 9, pp. 834845, 2012.

[2] S. Mohammadi, A. Ghani, and S. H. Sedighy, "Direction-of-arrival estimation in conformal microstrip patch array antenna," IEEE Transactions on Antennas and Propagation, vol. 66, no. 1, pp. 511-515, Jan 2018

[3] H. Krim and M. Viberg, "Two decades of array signal processing research: the parametric approach," Signal Processing Magazine, IEEE, vol. 13, no. 4, pp. 67-94, 1996.

[4] R. Schmidt, "Multiple emitter location and signal parameter estimation," IEEE Transactions on Antennas and Propagation, vol. 34, no. 3, pp. 276-280, 1986.

[5] A. Kuchar, M. Tangemann, and E. Bonek, "A real-time DOA-based smart antenna processor," IEEE Transactions on Vehicular Technology, vol. 51, no. 6, pp. 1279-1293, Nov 2002.

[6] C. Qian, L. Huang, and H. C. So, "Improved unitary root-MUSIC for DOA estimation based on pseudo-noise resampling," IEEE Signal Processing Letters, vol. 21, no. 2, pp. 140-144, 2013.

[7] F. Yan, Y. Shen, and M. Jin, "Fast DOA estimation based on a split subspace decomposition on the array covariance matrix," Signal Processing, vol. 115, pp. 1-8, 2015.

[8] C. Zhou, Z. Shi, Y. Gu, and N. Goodman, "DOA estimation by covariance matrix sparse reconstruction of coprime array," in Acoustics, Speech and Signal Processing (ICASSP), 2015 IEEE International Conference on. IEEE, 2015.

[9] X. Wu, W. Zhu, and J. Yan, "A high-resolution DOA estimation method with a family of nonconvex penalties," IEEE Transactions on Vehicular Technology, vol. 67, no. 6, pp. 4925-4938, June 2018.

[10] F. Shu, Y. Qin, T. Liu, L. Gui, Y. Zhang, J. Li, and Z. Han, "LowComplexity and High-Resolution DOA Estimation for Hybrid Analog and Digital Massive MIMO Receive Array," IEEE Transactions on Communications, vol. 66, no. 6, pp. 2487-2501, June 2018.

[11] Y. Wu, H. So, C. Hou, and J. Li, "Passive localization of near-field sources with a polarization sensitive array," IEEE Transactions on Antennas and Propagation, vol. 55, no. 8, pp. 2402-2408, 2007.

[12] W.-J. Zeng, X.-L. Li, H. Zou, and X.-D. Zhang, "Near-field multiple source localization using joint diagonalization," Signal Processing, vol. 89, no. 2, pp. 232-238, 2009.

[13] J. Xie, H. Tao, X. Rao, and J. Su, "Comments on "Near-field source localization via symmetric subarrays"," IEEE Signal Processing Letters, vol. 22, no. 5, pp. 643-644, May 2015.

[14] W. Zuo, J. Xin, W. Liu, N. Zheng, H. Ohmori, and A. Sano, "Localization of near-field sources based on linear prediction and oblique projection operator," IEEE Transactions on Signal Processing, vol. 67, no. 2, pp. 415-430, Jan 2019.

[15] W. Zuo, J. Xin, H. Ohmori, N. Zheng, and A. Sano, "Subspace-based algorithms for localization and tracking of multiple near-field sources," IEEE Journal of Selected Topics in Signal Processing, vol. 13, no. 1, pp. 156-171, March 2019.

[16] E. Grosicki, K. Abed-Meraim, and Y. Hua, "A weighted linear prediction method for near-field source localization," IEEE Transactions on Signal Processing, vol. 53, no. 10, pp. 3651-3660, 2005.

[17] W. Zhi and M. Chia, "Near-field source localization via symmetric subarrays," IEEE Signal Processing Letters, vol. 14, no. 6, pp. 409 412, 2007.

[18] M. Diao and S. Miao, "New method of parameter matching for 2D ESPRIT algorithms," Systems Engineering and Electronics, vol. 29, no. 8, pp. 1226-1229, 2007.

[19] B. Wang, J. Liu, and X. Sun, "Mixed sources localization based on sparse signal reconstruction," Signal Processing Letters, IEEE, vol. 19, no. 8, pp. 487-490, 2012.

[20] Z. Zheng, M. Fu, W.-Q. Wang, and H. C. So, "Mixed far-field and near-field source localization based on subarray cross-cumulant ," Signal Processing, 2018.

[21] Y. Huang and M. Barkat, "Near-field multiple source localization by passive sensor array," IEEE Transactions on Antennas and Propagation, vol. 39, no. 7, pp. 968-975, 1991.

[22] R. Challa and S. Shamsunder, "High-order subspace-based algorithms for passive localization of near-field sources," in Signals, Systems and Computers, 1995 Conference Record of the Twenty-Ninth Asilomar Conference on. IEEE, 1995.
[23] M. Haardt, R. Challa, and S. Shamsunder, "Improved bearing and range estimation via high-order subspace based unitary ESPRIT," in Signals, Systems and Computers, 1996. Conference Record of the Thirtieth Asilomar Conference on, vol. 1. IEEE, 1996.

[24] H. He, Y. Wang, and J. Saillard, "A high resolution method of source localization in near-field by using focusing technique," in Signal Processing Conference, 2008 16th European. IEEE, 2008.

[25] J. Liang, X. Zeng, B. Ji, J. Zhang, and F. Zhao, "A computationally efficient algorithm for joint range-DOA-frequency estimation of nearfield sources," Digital Signal Processing, vol. 19, no. 4, pp. 596-611, 2009.

[26] J. He, M. Swamy, and M. Ahmad, "Efficient application of MUSIC algorithm under the coexistence of far-field and near-field sources," IEEE Transactions on Signal Processing, vol. 60, no. 4, pp. 2066-2070, 2012.

[27] W. Zuo, J. Xin, N. Zheng, and A. Sano, "Subspace-based localization of far-field and near-field signals without eigendecomposition," IEEE Transactions on Signal Processing, vol. 66, no. 17, pp. 4461-4476, 2018.

[28] J. Liang and D. Liu, "Passive localization of near-field sources using cumulant," IEEE Sensors Journal, vol. 9, no. 8, pp. 953-960, Aug 2009.

[29] _ "Passive localization of mixed near-field and far-field sources using two-stage MUSIC algorithm," IEEE Transactions on Signal Processing, vol. 58, no. 1, pp. 108-120, 2010.

[30] B. Wang, Y. Zhao, and J. Liu, "Mixed-order MUSIC algorithm for localization of far-field and near-field sources," IEEE Signal Processing Letters, vol. 20, pp. 311-314, 2013.

[31] K. Wang, L. Wang, J. R. Shang, and X. X. Qu, "Mixed near-field and far-field source localization based on uniform linear array partition," IEEE Sensors Journal, vol. 16, no. 22, pp. 8083-8090, 2016.

[32] J. Li, Y. Wang, C. Le Bastard, G. Wei, B. Ma, S. Meng, and Z. Yu, "Simplified high-order DOA and range estimation with linear antenna array," IEEE Communications Letters, vol. 21, no. 1, pp. 76-79, 2017.

[33] B. Friedlander, "The root-MUSIC algorithm for direction finding with interpolated arrays," Signal Processing, vol. 30, no. 1, pp. 15-29, 1993.

[34] J. M. Mendel, "Tutorial on higher-order statistics (spectra) in signal processing and system theory: theoretical results and some applications," Proceedings of the IEEE, vol. 79, no. 3, pp. 278-305, 1991.

[35] K. Han and A. Nehorai, "Improved source number detection and direction estimation with nested arrays and ulas using jackknifing," IEEE Transactions on Signal Processing, vol. 61, no. 23, pp. 6118-6128, Dec 2013.

[36] M. Wax and T. Kailath, "Detection of signals by information theoretic criteria," IEEE Transactions on Acoustics, Speech, and Signal Processing, vol. 33, no. 2, pp. 387-392, April 1985.

[37] R. K. Olsson and L. K. Hansen, "Estimating the number of sources in a noisy convolutive mixture using BIC," in International Conference on Independent Component Analysis and Signal Separation. Springer, 2004, pp. 618-625.

[38] N. Yuen and B. Friedlander, "Performance analysis of higher order ESPRIT for localization of near-field sources," IEEE Transactions on Signal Processing, vol. 46, no. 3, pp. 709-719, 2002. 